\title{
Proving Failure of Queries for Definite Logic Programs Using XSB-Prolog
}

\author{
Nikolay Pelov and Maurice Bruynooghe \\ Departement Computerwetenschappen \\ Katholieke Universiteit Leuven \\ Celestijnenlaan $200 \mathrm{~A}$ \\ B-3001 Heverlee, Belgium \\ E-mail: \{pelov, maurice\}@cs.kuleuven.ac.be
}

\begin{abstract}
Proving failure of queries for definite logic programs can be done by constructing a finite model of the program in which the query is false. A general purpose model generator for first order logic can be used for this. A recent paper presented at PLILP98 shows how the peculiarities of definite programs can be exploited to obtain a better solution. There a procedure is described which combines abduction with tabulation and uses a meta-interpreter for heuristic control of the search. The current paper shows how similar results can be obtained by direct execution under the standard tabulation of the XSB-Prolog system. The loss of control is compensated for by better intelligent backtracking and more accurate failure analysis.
\end{abstract}

\section{Introduction}

In [2] methods are studied for proving that a query for a definite logic program fails. The general idea underlying all methods is the generation of a finite model of the definite program in which the query is false. However the approach developed in [2] is quite different from that used in general purpose model generators for first order logic such as FINDER [10], SEM [12], and $\mathrm{FMC}_{A T I N F}$ [7]. Whereas the latter systems search for a model in the space of interpretations, the former searches in the smaller space of pre-interpretations and applies a topdown proof procedure using tabulation to verify whether the query is false in the least model of the Horn theory based on the candidate pre-interpretation. Experiments in [3], an extended version of [2], show that the abductive procedure of [2] extended with intelligent backtracking [1] outperforms FINDER and $\mathrm{FMC}_{A T I N F}$ on problems where there are a large number of different interpretations for a given pre-interpretation. The difference is not only in number of backtracks, but also for some problems in time, and this notwithstanding the former is implemented as a straightforward meta-interpreter in Prolog while the latter are sophisticated implementations in a more low level language.

The current paper describes how the meta-interpreter can be replaced by a more direct implementation in XSB-Prolog [9, 4] which relies on the XSB system to perform the tabulation. This is not a straightforward task because 
of the intelligent backtracking and because the meta-interpreter does not follow the standard depth-first left-to-right search strategy but uses heuristics to direct the search towards early failures and selects the pre-interpretation on the fly, as components are needed by the proof procedure. To exploit the tabling system underlying XSB, one has to stick to the depth-first left-to-right execution order and one should not modify the program by creating new components of the pre-interpretation while evaluating a call to a tabled predicate.

The random selection of an initial pre-interpretation, combined with the loss of control over the search results in a system which has to explore a substantially larger part of the search space than the original system. The paper introduces two innovations to compensate for this. Firstly, it uses a variant of intelligent backtracking which is much less dependent on the random initial order of the choice points. Secondly, it introduces a more accurate failure analysis, so that smaller conflict sets are obtained and that the intelligent backtracking selects its targets with more accuracy.

The motivation for this research is in the world of planning. Planners are typically programs which search in an infinite space of candidate plans for a plan satisfying all requirements. The planner searches forever (until some resource is exhausted) when no candidate plan satisfies all requirements. Hence it useful to have methods to show that the problem has no solution. It turns out that our approach outperforms first order model generators on planning problems.

In the next section we recall some basic notions about semantic of definite logic programs. In Section 3 we describe our approach in more detail and then in Section 4 we show the results of testing our system on different problems. The comparison not only includes the model generator FINDER [10] as in [2], and FMC $_{A T I N F}$ as in [3] but also SEM [12].

\section{Preliminaries}

Now we will recall some basic definitions about semantics of definite programs. Most of them are taken from [6].

A pre-interpretation $J$ of a program $P$ consists of domain $D=\left\{d_{1}, \ldots, d_{m}\right\}^{1}$ and for each $n$-ary function symbol $f$ in $P$ a mapping $f_{J}$ from $D^{n}$ to $D$. Following the literature on model generators, a term of the form $f\left(d_{1}, \ldots, d_{n}\right)$ where $d_{1}, \ldots, d_{n} \in D$ is called a cell. Given a program $P$ and domain size $m$, the set of all cells is fixed. A pair $\langle c, v\rangle$ where $c$ is a cell and $v \in D$ is the mapping of that cell is called a component and $v$ the value of the component. A set of components defines a pre-interpretation if there is exactly one component $\langle c, v\rangle$ for each cell.

A variable assignment $V$ wrt. expression $E$ and pre-interpretation $J$ consists of an assignment of an element in the domain $D$ for each variable in $E$. A term assignment wrt. $J$ and $V$ is defined as follows: each variable is given its assignment according to $V$; each constant is given its assignment according to $J$; if $d_{1}, \ldots, d_{n}$ are the term assignments of $t_{1}, \ldots, t_{n}$ then the assignment of $f\left(t_{1}, \ldots, t_{n}\right)$ is the value of the cell $f\left(d_{1}, \ldots, d_{n}\right)$.

\footnotetext{
${ }^{1}$ We will consider only domains with finite size.
} 
An interpretation $I$ based on a pre-interpretation $J$ consists of a mapping $p_{I}$ from $D^{n}$ to $\{$ false,true $\}$ for every $n$-ary predicate $p$ in $P$. An interpretation $I$ is often defined as the set of atoms $p\left(d_{1}, \ldots, d_{n}\right)$ for which $p\left(d_{1}, \ldots, d_{n}\right)$ is mapped to true. An interpretation $M$ is a model of a program $P$ iff all clauses in $P$ are true in $M$. For definite program, the intersection of two models is also a model hence a definite program always has a unique least model. As a consequence, if a conjunction of atoms is false in some model then it is also false in the least model of a definite program.

Throughout the paper we will use the following simple example about even and odd numbers to show the different concepts and program transformations.

even (zero).

even $(s(X)):-\operatorname{odd}(X)$.

$\operatorname{odd}(s(X)):-\operatorname{even}(X)$.

Consider a query ?- even $(X)$, odd $(X)$. For simplicity of the presentation we will add to the program the definite clause

even_odd :- even $(X), \operatorname{odd}(X)$.

and consider the query ?- even_odd. It cannot succeed as ?- even_odd is not a logical consequence of the program. The SLD proof procedure does not terminate. This is still the case when extended with tabulation as in XSB-Prolog.

We choose a domain with two elements $D=\{0,1\}$ and consider the preinterpretation $J=\left\{\right.$ zero $\left._{J}=0, s_{J}(0)=1, s_{J}(1)=0\right\}$. The least model of the definite program is $\{$ even $(0)$,odd(1) $\}$ and the atom even_odd is false in this model.

\section{The Method}

Figure 1 shows the general architecture of the system. The input consists of a definite program $P$, a query ? $Q Q$ and domain size $m$. First the program and the query are transformed to $P^{t}$ and ?- $Q^{t}$. The transformation replaces all functional symbols with calls to predicates defining the components of the preinterpretation and allows the program to collect the components which were used during the evaluation of the query. Also an initial pre-interpretation $J$ is constructed for the given domain size $m$. Then the query ?- $Q^{t}$ is evaluated wrt. the program $P^{t}$ and the current pre-interpretation $J$. If the query succeeds then it also returns a set of components $C S$ which are necessary for the success of the proof. Then, based on $C S$, the pre-interpretation is modified and the query is run again. If we have exhausted all possible pre-interpretations for the given domain size then we can eventually increase it and run the system again. If the query ?- $Q^{t}$ fails then $Q^{t}$ is false in the least model based on the pre-interpretation $J$ and we can conclude that the original query ?- $Q$ cannot succeed. 


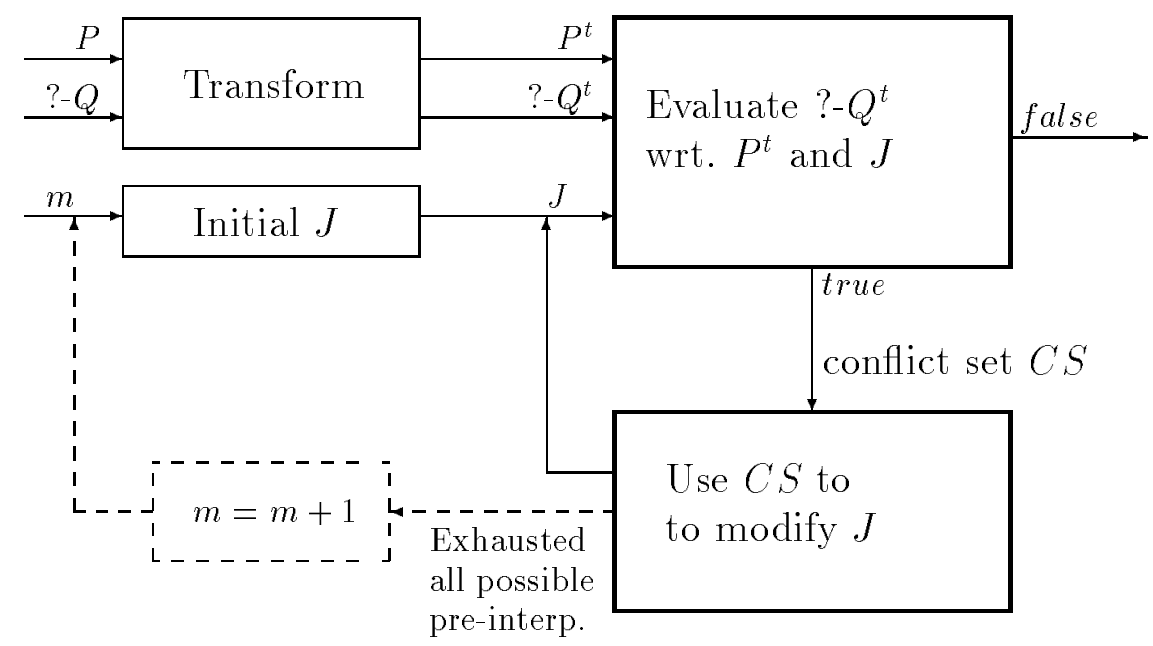

Fig. 1. System architecture

\subsection{Basic Transformation}

To evaluate the query in the least model based on a pre-interpretation $J$, we use a variant of the abstract compilation approach to program analysis used by Codish and Demoen in [5]. The pre-interpretation $J$ of a $n$-ary function $f$ is represented by a set of facts $p_{f}\left(d_{1}, \ldots, d_{n}, v\right)$; one fact for each cell $f\left(d_{1}, \ldots, d_{n}\right)$. In the source program, non variable terms are represented by their pre-interpretation. This is achieved by replacing a term $f\left(t_{1}, \ldots, t_{n}\right)$ by a fresh variable $X$ and introducing a call $p_{f}\left(t_{1}, \ldots, t_{n}, X\right)$. This transformation is repeated for the non variable terms in $t_{1}, \ldots, t_{n}$ until all functions are eliminated. Codish and Demoen evaluate the resulting DATALOG program bottom up, obtaining the least model which expresses declarative properties of the program. In [2], one also transforms the query and using a top-down procedure with tabulation checks whether it fails. Experience showed that one typically ends up with computing the whole model of the predicates reachable from the query. So the meta-interpreter used there tables only the most general call for each predicate. As we want direct execution under XSB, our transformation has to take care that a program predicate is only called with all variables free and different, so that XSB tables only the most general call. To achieve this, a predicate $p_{f}(\ldots)$ which is added to compute a term $t$ in a call is inserted after the call and a predicate which is added to compute a term in the head is inserted at the end of the clause. Finally, when a call to a program predicate contains a variable $X$ which already occurs to the left of its position in the clause, then it is replaced by a fresh variable $Y$ and an equality $X=Y$ is inserted after the call. The calls to the pre-interpretation are not tabled, and a call $p_{f}(g(\ldots), \ldots)$ is transformed in $p_{g}(\ldots, X), p_{f}(X, \ldots)$. This 
gives less branching than when $p_{g}(\ldots)$ is added after $p_{f}(\ldots)$. For our example this gives the following code:

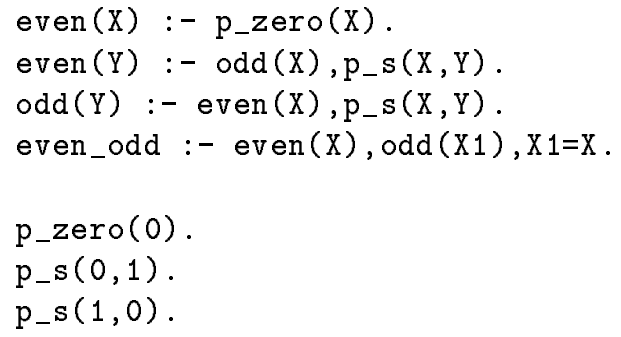

In [2], values are assigned to the cells of the pre-interpretation in an abductive way, as needed by the heuristic search for a proof of the query. When a proof is found, standard backtracking occurs: the last assigned value is modified. To have direct execution under XSB, the pre-interpretation has to be fixed in advance. Obviously, it is not feasible to enumerate all possible pre-interpretations until one is found for which the query fails. The search has to be guided by the proof found so far. Failure analysis and intelligent backtracking have to be incorporated to obtain a usable system.

\subsection{Failure Analysis}

Elementary Failure Analysis. As the goal is to find a pre-interpretation for which the query fails, failure occurs when the query succeeds. In the more general setting of first order model generation, failure occurs when some formula gets the wrong truth value. The FINDER and $\mathrm{FMC}_{A T I N F}$ systems keep track of which cells are used in evaluating a formula and when the formula receives the wrong truth value, the set of cells used in evaluating it is used to direct the backtracking. In [3] the meta-interpreter is extended with such a failure analysis and intelligent backtracking is used to guide the search. This substantially improved the performance of the system. Incorporating these features in the current approach which relies on direct execution with XSB of the transformed query, requires special care. First let us formalize the notion of conflict set (refutation in first order model generators [7, 10]).

Definition 1 (Conflict set). A conflict set $C S$ of a definite program $P$ and query $Q$ is a finite set of components such that for any pre-interpretation $J$ for which $C S \subseteq J$ follows that $Q$ is true in any model of $P$ based on $J$.

The idea is that any pre-interpretation $J$ which has the same values for all components from the conflict set $C S$ can not be extended to an interpretation in which the query fails. Hence any candidate pre-interpretation must differ from $C S$ in the value of at least one component. Exploiting conflict sets requires first to compute them. This can be done by adding to the program predicates an extra argument which is used to collect the components used for solving a call to this predicate. For example a call even $(X)$ is replaced by even $(X, C S)$ and the answer 
even (0) becomes even ( $0,[$ pzero(0)]). However there is a potential problem. Also even $\left(0,\left[p_{z} z e r o(0), p_{s} s(0,1), p_{s} s(1,0)\right]\right)$ is an answer. Previously, the tabling system did not recognize it as a new answer and did not use it to solve calls to even/1. But as the value of the added second argument differs from that in the first answer, XSB will also use it to solve calls to even $/ 2$ and it will obtain a third answer. Fortunately, if the list of used components is reduced to some canonical form, then the third answer will be identical to the second and the evaluation will terminate. However, this repetition of answers with different lists of components can substantially increase the cost of the query evaluation. Fortunately the XSB system has built-in predicates to inspect and modify the tables so we can control this behavior. The idea is to replace a clause

$$
\mathrm{p}(\mathrm{X}, \mathrm{CS}):- \text { Body }
$$

with a clause

$$
\mathrm{p}(\mathrm{X}, \mathrm{CS}):- \text { Body, check_return }(\mathrm{p}(\mathrm{X}, \mathrm{CS})) \text {. }
$$

When the body of the clause succeeds, XSB will process the answer $p(X, C S)$ (add it to the table for the call to $p / 2$ if it is new). Remember, that as the transformed program makes only most general calls there is only one table associated with each predicate. Using the built-ins, the predicate check_return/1 looks up the previous answers in the table for $p / 2$ and compares them with the candidate answer $p(X, C S)$. If there is no other answer with the same $X$ then check_return $/ 1$ and thus $p / 2$ simply succeed. The interesting case is when the table already holds an answer $p\left(X, C S_{\text {old }}\right)$ with a different conflict set $C S_{\text {old }}$ (if $C S_{\text {old }}=C S$ then XSB will recognize it is a duplicate answer). Then several strategies are possible for check_return/1:

- The simplest approach is to let check_return/1 fail when the table already holds an answer with the same $X$.

- An alternative approach is to check whether the new conflict set $C S$ is "better" than $C S_{\text {old }}$. Then the old answer is removed from the table and check_return/1 succeeds. Otherwise check_return/1 fails.

- Finally, but more expensive for the overall query evaluation, one could allow several answers, only rejecting/removing redundant ones $\left(p\left(X, C S_{1}\right)\right.$ is redundant wrt. $p\left(X, C S_{2}\right)$ if $\left.C S_{1} \supseteq C S_{2}\right)$.

Advanced Failure Analysis. A conflict set can be called minimal if it has no subset which is a conflict set. Obviously it is not feasible to compute minimal conflict sets. However, simply collecting the components used in a proof can be a large overestimation. For example, in our planning problems, a three argument predicate is used: one argument is the initial state, one argument is the final state and one argument is the description of the derived plan. The pre-interpretation of the terms representing the plan is completely irrelevant for the failure of the query. However the components used to compute it will be part of the conflict set. 
To see how to refine our failure analysis, let us reconsider how answers are obtained. Using a slightly different notation, the base case of the even/1 predicate can be written as:

even $(\mathrm{X}):-\mathrm{X}=0_{-} \mathrm{J}$

This represents the basic answer, parameterized by the pre-interpretation $J$. Now consider the definition of the odd/1 predicate:

$\operatorname{odd}(X):-\operatorname{even}(Y), X=s_{-} J(Y)$

An answer of odd/1 is obtained by performing resolution with the basic answer for even/1, yielding:

$\operatorname{odd}(\mathrm{X}):-\mathrm{Y}=\mathrm{X} 1, \mathrm{X} 1=\mathrm{O}_{-} \mathrm{J}, \mathrm{X}=\mathrm{S}_{-} \mathrm{J}(\mathrm{Y})$.

This can be generalized, answers for a predicate $p / n$ are of the form:

$$
p\left(X_{1}, \ldots, X_{n}\right) \leftarrow X_{1}=t_{1 J}, \ldots, X_{N}=t_{n_{J}}, E q s
$$

with Eqs a set of equations involving $X_{1}, \ldots, X_{n}$ and some local variables $Y_{1}, \ldots, Y_{n}$. Under the elementary failure analysis the answer is $p\left(t_{1_{J}}, \ldots, t_{n_{J}}\right)$ and the associated conflict set is the set of components used in computing $t_{1}, \ldots, t_{n_{l}}$ and the terms of Eqs.

The basis for the advanced failure analysis is the observation that the answer clauses can be simplified while preserving the solution they represent. Terms form equivalence classes under a pre-interpretations. Members of the equivalence class can be represented by the domain element which is their pre-interpretation and equalities between terms modulo equivalence class can be simplified using three of the four Martelli-Montanari simplification rules:

$-p\left(t_{1}, \ldots, t_{n_{J}}\right) \leftarrow X=X, E q s$ is equivalent to $p\left(t_{1}, \ldots, t_{n_{J}}\right) \leftarrow E q s$ (remove)

$-p\left(t_{1_{J}}, \ldots, t_{n_{J}}\right) \leftarrow t_{J}=X$, Eqs is equivalent to $p\left(t_{1_{J}}, \ldots, t_{n_{J}}\right) \leftarrow X=t_{J}$, Eqs (switch)

$-p\left(t_{1}, \ldots, t_{n_{J}}\right) \leftarrow X=t_{J}$, Eqs is equivalent to $p\left(t_{1_{J}}, \ldots, t_{n_{J}}\right)\left\{X / t_{J}\right\} \leftarrow E q s\left\{X / t_{J}\right\}$ (substitute)

Note that $f_{J}\left(t_{1_{J}}, \ldots, t_{n_{J}}\right)=g_{J}\left(s_{1_{J}}, \ldots, s_{m_{J}}\right), E q s$ is not equivalent to false and that $f_{J}\left(t_{1_{J}}, \ldots, t_{n_{J}}\right)=f_{J}\left(s_{1_{J}}, \ldots, s_{n_{J}}\right), E q s$ is not equivalent to $t_{1_{J}}=$ $s_{1}, \ldots, t_{n_{J}}=s_{n_{J}}, E q s$, hence peel is not allowed.

So an answer can be simplified to a form

$$
p\left(t_{1 J}, \ldots, t_{n_{J}}\right) \leftarrow E q s
$$

where Eqs contains equations between non variable terms and some of the $t_{i_{j}}$ in the head can be variables. The pre-interpretations in the terms of Eqs decide whether Eqs is interpreted as true or false, hence the components used in interpreting the terms in Eqs form the real conflict set of the answer. However 
also the components used to interpret the terms $t_{i_{J}}$ of the head are important. When the answer is used to solve a call, they become part of new equations. Hence, with each variable we should associate a set holding the components used in evaluating the term the variable is bound to and with each answer we should associate the "real" conflict set. Moreover, the execution of the equalities $X=Y$ has to be monitored. When one of $X$ or $Y$ is free then unification can be performed, otherwise if $X$ and $Y$ have the same interpretation then the sets of components associated with $X$ and $Y$ have to be added to the conflict set of the answer (as before the equality fails when $X$ and $Y$ have a different interpretation). Note that our transformation is such that calls have fresh variables as arguments, so the equality between an argument of a call and an argument of an answer always involves a free variable and is correctly handled by standard unification. A final point is that the body of the compiled clause have to be carefully ordered: equalities on predicate calls involving a variable $X$ should precede the interpretation of a term containing $X$, e.g. $p(X), Y=f_{J}(X)$ is a correct ordering: first the call $p / 1$ binds $X$ to a domain element and also returns the set of components $C S_{X}$ used in computing that domain element. Then $Y$ is bound to a domain element and the set of components used in computing it is $\left\{f_{J}(X)\right\} \cup C S_{X}$. Taking the above into account, the code for our example is as follows:

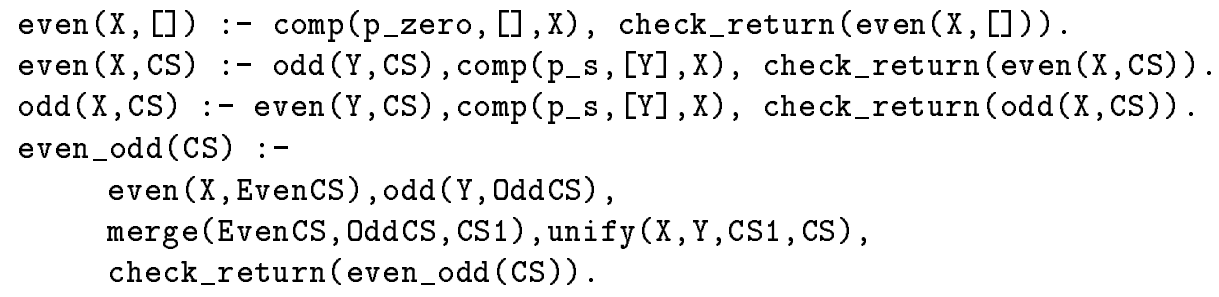

Calls to the pre-interpretation are made through an intermediate predicate comp/3 defined below. The call to combine_arg_cs $/ 3$ collects the conflict sets associated with the ground arguments of the function to be interpreted (none if the argument is a free variable) in ArgsCS and merge/3 extends ArgsCS with Comp, the consulted component of the pre-interpretation, to obtain the final conflict set ResCS.

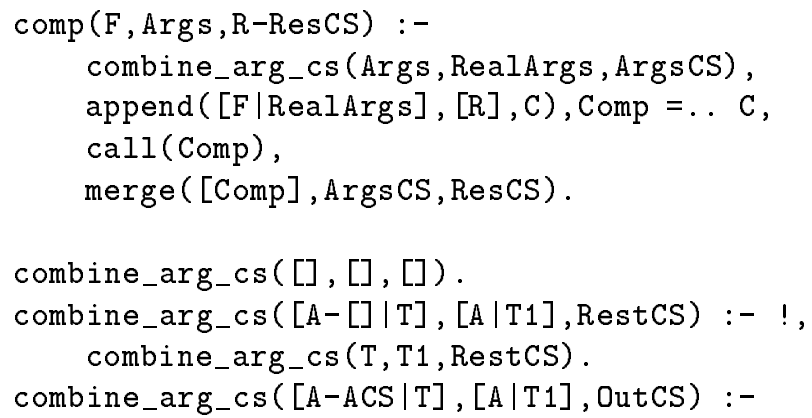




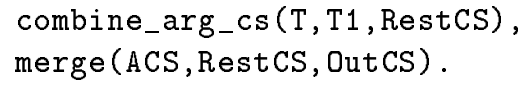

The merge/3 predicate makes the union of two sets (represented as lists) and places the result in a canonical form and unify/4 is used to monitor the unification process and can be defined by the following Prolog code:

unify $(X, Y, S, S):-(\operatorname{var}(X) ; \operatorname{var}(Y)), \quad, \quad X=Y$.

unify $(X-S x, X-S y, S i n, S o u t):-\operatorname{merge}(S x, S y, S)$, merge(S,Sin, Sout).

The first two arguments are the terms to be unified, the third is the current conflict set of the clause and the last argument is the new conflict set of the clause. The first clause handles the case that one is a free variable: unification is performed and the conflict set of the clause remains the same. The second clause handles the case that both arguments $X$ and $Y$ are bound to the same domain element. The set of components used in evaluating the first argument $(S \boldsymbol{x})$ and in evaluating the second argument $(S y)$ are added to Sin yielding Sout.

\subsection{Intelligent Backtracking}

Under standard backtracking, candidate pre-interpretations are enumerated according to some fixed total ordering $c_{1}, c_{2}, \ldots, c_{n}$ of the cells. When some partial solution $c_{1}=d_{1}^{1}, c_{2}=d_{2}^{1}, \ldots, c_{m}=d_{m}^{1}$ is rejected then the value assignment $d_{m}^{1}$ for the last cell $c_{n}$ is modified. If no other value is left, then $c_{m-1}$ is modified (and all domain elements become again available for $c_{m}$ ). The simplest use of conflict sets is based on the observation that no extension of the conflict set can be a solution, so the last element according to the total order over the cells of the conflict set is selected and the assignment to this cell is modified. However also secondary conflict sets can be derived [1]. Assume, due to different conflicts, all values for some cell $c_{n}$ have been rejected. With $\left\{c_{i, 1}, \ldots, c_{i, k_{i}}, c_{n}\right\}$ the conflict set which led the rejection of $d_{i}$ we can formalize the knowledge in the conflict sets as:

$$
\begin{aligned}
& c_{1,1}=d_{1,1} \wedge \ldots \wedge c_{1, k_{1}}=d_{1, k_{1}} \wedge c_{n}=d_{1} \rightarrow \text { false } \\
& \vdots \\
& c_{m, 1}=d_{m, 1} \wedge \ldots \wedge c_{m, k_{m}}=d_{m, k_{m}} \wedge c_{n}=d_{m} \rightarrow \text { false } .
\end{aligned}
$$

As we have that cell $c_{n}$ must be assigned some domain element, we have $c_{n}=$ $d_{1} \vee \ldots \vee c_{n}=d_{m}$. Applying hyper-resolution [8], one can infer

$$
\begin{aligned}
& c_{1,1}=d_{1,1} \wedge \ldots \wedge c_{1, k_{1}}=d_{1, k_{1}} \wedge \\
& \vdots \\
& c_{m, 1}=d_{m, 1} \wedge \ldots \wedge c_{m, k_{m}}=d_{m, k_{m}} \rightarrow \text { false }
\end{aligned}
$$

which says that $\left\{c_{1,1}, \ldots, c_{1, k_{1}}, \ldots, c_{m, 1}, \ldots, c_{m, k_{m}}\right\}$ is also a conflict set. 
At the implementation level, an accumulated conflict set is associated with each cell and initialized as empty. When a conflict $\left\{c_{1}, \ldots, c_{n-1}, c_{n}\right\}$ is derived with $c_{n}$ its last cell, then $\left\{c_{1}, \ldots, c_{n-1}\right\}$ is added to the accumulated conflict set of $c_{n}$. Once all assignments to a cell are exhausted, its associated conflict set holds the secondary conflict which can be used to direct further backtracking. This is the approach taken in [3] where it worked quite well, as the initial order was carefully chosen. In the current implementation, where the initial order over the cells is random, the system had to do much more search before finding a solution. Hence we adopted a variant of intelligent backtracking mentioned in [1] which leaves the cells unordered until they participate in a conflict. Under this approach, cells are split over two sets, a set with a total order (initially empty) and a set which is unordered. When a conflict is found, the cells from it which are in the unordered set (if any) are moved to the end of the ordered set. Then the last cell of the conflict set is chosen as target of the backtracking. Cells which are after the target in the total order return to the unordered set. This approach resulted in substantially better results.

\subsection{Dealing with Equational Problems}

There exists many problems which contain only one predicate, the equality predicate $e q / 2$. They consist of a number of facts $e q\left(t_{i_{1}}, t_{i_{2}}\right) \leftarrow$ for $i=1, \ldots, m$ and a number of denials $\leftarrow e q\left(s_{j_{1}}, s_{j_{2}}\right)$ for $j=1, \ldots, n$. To solve such problems, one has to add to the program the axioms for the equality theory for reflexivity, symmetry, transitivity and function substitution, the latter consists of an axiom

$$
f\left(X_{1}, \ldots, X_{n}\right)=f\left(Y_{1}, \ldots, Y_{n}\right) \leftarrow X_{1}=Y_{1} \wedge \ldots \wedge X_{n}=Y_{n} .
$$

for each functor $f / n$. The least model of the standard equality theory is the identity relation over the domain of the interpretation, hence the search space can be reduced by restricting the interpretation of $e q / 2$ to the identity relation.

In the abductive system of [3], this is achieved by initializing the interpretation of $e q / 2$ as identity, and removing the standard equality theory (only the problem specific facts and denials remain). Backtracking is initiated as soon as either one of the denials $e q\left(s_{j_{1}}, s_{j_{2}}\right)$ evaluates to true or one of the facts $e q\left(t_{i_{1}}, t_{i_{2}}\right)$ results in an answer which is not in the identity relation.

With direct execution under XSB, a slightly different approach is required. Unification reduces to the identity relation, hence after compiling the terms, the call to $e q / 2$ can be done by unifying the compiled terms. However, the problem is that all facts and denials need to be activated. Therefore a new predicate $p / 0$ is introduced and defined as follows:

$$
\begin{array}{ll}
p \leftarrow \neg e q\left(t_{i_{1}}, t_{i_{2}}\right) . & i=1, \ldots, m \\
p \leftarrow e q\left(s_{j_{1}}, s_{j_{2}}\right) . & j=1, \ldots, n
\end{array}
$$

Proving failure of the query $\leftarrow p$ yields the desired pre-interpretation. Indeed $p$ is equivalent to

$$
p \leftarrow \bigvee_{1 \leq i \leq m} \exists \neg e q\left(t_{i_{1}}, t_{i_{2}}\right) \vee \bigvee_{1 \leq j \leq n} \exists e q\left(s_{j_{1}}, s_{j_{2}}\right)
$$


Hence $p$ fails if the right-hand side is true, i.e. if

$$
\bigwedge_{1 \leq i \leq m} \forall e q\left(t_{i_{1}}, t_{i_{2}}\right) \wedge \bigwedge_{1 \leq j \leq n} \forall \neg e q\left(s_{j_{1}}, s_{j_{2}}\right)
$$

is true. $\forall e q\left(t_{i_{1}}, t_{i_{2}}\right)$ is equivalent with the fact $e q\left(t_{i_{1}}, t_{i_{2}}\right)$ and $\forall \neg e q\left(s_{j_{1}}, s_{j_{2}}\right)$ is equivalent to the denial $\leftarrow e q\left(s_{j_{1}}, s_{j_{2}}\right)$. Thus $p$ fails if the conjunction of the original facts and denials is true under the chosen pre-interpretation. Compilation of terms is as described in Section 3.1, i.e. a call $e q\left(s_{j_{1}}, s_{j_{2}}\right)$ is replaced by a call $X_{j_{1}}=X_{j_{2}}$ preceded by the code computing the pre-interpretation of $s_{j_{1}}$ and $s_{j_{2}}$. A call $\neg e q\left(t_{i_{1}}, t_{i_{2}}\right)$ is handled in a similar way; the built-in $\backslash=$ (not unifiable) can be used instead of not equal. However, special care is required to ensure the arguments are ground in case $t_{i_{1}}$ or $t_{i_{2}}$ is a variable. Whereas the compilation leaves such variables intact, here it has to be mapped (the mapping introduces a backtrack point) to a domain element.

Similarly as in Section 3.2, conflict sets can be associated with terms for the task of advanced failure analysis. Hence a call $\neg e q\left(t_{i_{1}}, t_{i_{2}}\right)$ is transformed in the sequence interpret $\left(t_{i_{1}}, X_{i_{1}}\right)$, interpret $\left(t_{i_{2}}, X_{i_{2}}\right)$, disunify $\left(X_{i_{1}}, X_{i_{2}}, S_{i n}, S_{\text {out }}\right)$ where interpret/2 is an abbreviation for the sequence of calls computing the preinterpretation of the term and the associated conflict set and disunify/4 is defined as

disunify (X-Sx, Y-Sy, Sin, Sout) :-

$X \backslash=Y$, merge $(S x, S y, S)$, merge(S,Sin, Sout).

\section{Experiments}

\subsection{The Problems}

We tested our system with a large number of different problems. Below we give a short description for each one of them and for some of them the source code is given in Appendix A.

List Manipulation. The appendlast problem uses the standard definition of the predicates append and last and the following query:

appendlast :- $\operatorname{append}(\mathrm{X},[\mathrm{a}], \mathrm{Xs}), \operatorname{last}(\mathrm{Xs}, \mathrm{b})$.

The reverselast problem is similar to the appendlast problem but uses the version of the predicate reverse with accumulator:

reverselast:- $\operatorname{reverse}(L, R,[a]), \operatorname{last}(R, b)$.

The nreverselast problem uses the "naive" definition of reverse:

nreverselast :- reverse $([a \mid X], R), \operatorname{last}(R, b)$. 
Multisets. The multiset?o are programs to check the equivalence of two multisets using a binary operator "o" to represent them. multiset3o is a problem which has a solution, thus failure cannot be proven for it.

Planning in the Blocks-World. These are simple problems for planning in the blocks-world. The theory for the blockpair problems has, besides the usual actions of the blocks-world, an action to add or remove a pair of blocks. In the blockzero problems, the extra action is to create a new block named $s(X)$ on top of a clear block $X$.

The queries ending in "o" use multisets based on the function $0 / 2$ and those ending in "l" use a standard list representation. Those problems which have the number 2 in their name do not collect the plan and those having 3 store the plan in the second argument. blockzero $21 \mathrm{~s}^{2}$ is a problem which has a solution.

TPTP-Problems. The rest of the examples are taken from the TPTP problem library [11]. In Table 1 in brackets are given the TPTP names for each one of them. All these problems are equational problems and are transformed in the way described in Section 3.4.

The tba problem is to prove an independence of one axiom for ternary boolean algebra.

The grp problem is to prove that some axiom is not a single axiom for group theory.

The c13 problem is from the domain of combinatory logic and the goal is to find a set of combinators which satisfy axioms $S$ and $W$ and do not satisfy the weak fixed point property.

Table 1 gives some details about the properties of the problems. The column \#pred shows the number of predicates. The column size dom gives the domain size for which the query has been evaluated (which is, for the failing queries, the minimum domain size for which a model proving failure exists). The column size pre gives the number of cells in the pre-interpretation and the next column \#pre gives the number of all possible pre-interpretations for the given domain size. The column size int gives the number of atoms to be assigned a truth value in an interpretation and the last column \#int/pre gives the number of different interpretations for a fixed pre-interpretation. For the TPTP problems this value is 1 because they have only one predicate for which the interpretation is known to be identity.

\subsection{Results}

The results with $\mathrm{FMC}_{A T I N F}$ were taken from [7] or were sent to us by its author which was using a SUN 4 ELC machine. All other systems were run on SUN Sparc Ultra-2 computer. The system AB is the abductive system described in

\footnotetext{
${ }^{2}$ corresponds to blocksol in [2] and [3]
} 
Table 1. Example properties

\begin{tabular}{|l|r|r|r|r|r|r|}
\hline Example & \#pred & size dom & size pre & \#pre & size int & \#int/pre \\
\hline appendlast & 2 & 3 & 12 & $3^{12}$ & 13 & $2^{13}$ \\
reverselast & 2 & 3 & 12 & $3^{12}$ & 13 & $2^{13}$ \\
nreverselast & 3 & 5 & 28 & $5^{28}$ & 150 & $2^{150}$ \\
\hline multiset1o & 1 & 2 & 7 & $2^{7}$ & 4 & $2^{4}$ \\
multiset2o & 1 & 2 & 7 & $2^{7}$ & 4 & $2^{4}$ \\
multiset3o & 1 & 2 & 7 & $2^{7}$ & 4 & $2^{4}$ \\
\hline blockpair2o & 3 & 2 & 19 & $2^{19}$ & 12 & $2^{12}$ \\
blockpair3o & 3 & 2 & 36 & $2^{36}$ & 20 & $2^{20}$ \\
blockpair21 & 5 & 2 & 19 & $2^{19}$ & 32 & $2^{32}$ \\
blockpair31 & 5 & 2 & 36 & $2^{36}$ & 40 & $2^{40}$ \\
blockzero2o & 3 & 2 & 19 & $2^{19}$ & 12 & $2^{12}$ \\
blockzero3o & 3 & 2 & 35 & $2^{35}$ & 20 & $2^{20}$ \\
blockzero2l & 5 & 2 & 19 & $2^{19}$ & 32 & $2^{32}$ \\
blockzero3l & 5 & 2 & 35 & $2^{35}$ & 40 & $2^{40}$ \\
blockzero2ls & 5 & 2 & 19 & $2^{19}$ & 32 & $2^{32}$ \\
\hline tba (BOO019-1) & 1 & 3 & 32 & $3^{32}$ & 9 & 1 \\
grp (GRP081-1) & 1 & 2 & 17 & $2^{17}$ & 4 & 1 \\
cl3 (COL005-1) & 1 & 3 & 12 & $3^{12}$ & 9 & 1 \\
\hline
\end{tabular}

[3], however, running under (the slower) XSB-Prolog instead of Master Prolog for equal comparison. We used FINDER [10] version 3.0.2 and SEM [12] version 1.7 which are well known model generators implemented in $\mathrm{C}$.

The system naive results from the direct translation of the system AB to XSB: it uses the same failure analysis, it starts from a random total order over the cells of the pre-interpretation and it uses the simplest variant of checkreturn which sticks to the first answer whatever the associated conflict set is. For the TPTP problems the standard equality axioms were used.

The systems single $C S$ and best $C S$ use a more sophisticated version of check_return which prefers the answer with the shorter conflict set, advanced failure analysis and the more sophisticated version of intelligent backtracking which leaves elements unordered until they participate in a conflict set. The system single CS uses the first answer to the top level query to direct the backtracking. The system best $C S$ computes all answers to the top level query and then selects from them the conflict set which will add the fewest number of cells to the ordered sequence. Both systems use the technique described in Section 3.4 on the TPTP problems.

Table 2 gives the times obtained by the different systems. The time is in seconds unless followed by $\mathrm{H}$, then it is in hours. A "-" means the example was not run. A ">n" means the system had still no solution after time $n$.

Table 3 shows the number of generated and tested pre-interpretations (number of backtracks). For the SEM system, we have modified the source code to report exactly this number. For the FINDER system we report the sum of the 
Table 2. Execution times

\begin{tabular}{|l|r|r|r|r|r|r|r|}
\hline Example & naive & single CS & best CS & AB & FINDER & SEM & FMC $_{\text {ATINF }}$ \\
\hline appendlast & 919 & 0.76 & 1.63 & 1.42 & 0.07 & 0.01 & 45.21 \\
reverselast & 918 & 0.85 & 1.85 & 1.00 & 0.10 & 0.01 & 10.79 \\
nreverselast & $>2706$ & $>1673$ & 178 & $17.5 \mathrm{H}$ & $>1446$ & 957 & $>900$ \\
\hline multiseto1 & 0.18 & 0.06 & 0.12 & 0.08 & 0.02 & 0.01 & - \\
multiseto2 & 0.07 & 0.20 & 0.47 & 0.10 & 0.02 & 0.01 & 0.02 \\
multiseto3 & 0.94 & 0.54 & 2.77 & 0.28 & 0.03 & 0.01 & - \\
\hline blockpair2o & 451 & 0.86 & 3.14 & 5.05 & 0.07 & 0.05 & 7.31 \\
blockpair3o & $>58$ & 0.94 & 3.90 & 21.97 & 0.18 & 0.23 & $>900$ \\
blockpair21 & 5303 & 1.86 & 7.85 & 3.56 & 0.04 & 0.05 & 204.9 \\
blockpair31 & $>222$ & 2.05 & 9.70 & 53.88 & 0.12 & 0.18 & $>900$ \\
blockzero20 & 7.93 & 7.94 & 4.35 & 2.84 & 0.11 & 0.09 & - \\
blockzero3o & 162 & 8.86 & 5.41 & 24.48 & 0.22 & 1.98 & - \\
blockzero2l & 18.49 & 2.00 & 20.71 & 5.67 & 0.23 & 0.10 & - \\
blockzero31 & 40.35 & 2.06 & 24.76 & 37.23 & 0.33 & 2.39 & - \\
blockzero2ls & $11.8 \mathrm{H}$ & 648 & 2631 & 593 & 2287 & 5.05 & $>900$ \\
\hline tba & $>950$ & 1331 & 3.65 & 3.29 & 0.03 & 0.03 & 0.06 \\
grp & 1189 & 1.05 & 5.89 & 13.94 & 0.03 & 0.01 & - \\
cl3 & 0.13 & 3.85 & 1.63 & 1.03 & 0.02 & 0.03 & 0.04 \\
\hline
\end{tabular}

number of bad candidates tested and other backtracks. Also in this table "_" means not run, ">n" means already $n$ backtracks when interrupted. For the system best $C S$ we give an additional column total which shows the total number of conflict sets obtained as "answers" to the query (divided by the number of backtracks, this gives the average number of conflict sets obtained when running the query).

\subsection{Discussion}

Comparing the systems naive and $\mathrm{AB}$, we see that the straightforward transfer of AB to XSB results in a much worse behavior. Hence the heuristics used by AB to control the search have a big impact.

The effect of the advanced failure analysis is not reported separately. Its impact is only visible in the block $* 3$ ? problems which compute, for the failure analysis, an irrelevant output argument. The advanced failure analysis makes these problems behave as well as the corresponding block*2? problems. Note that the AB system as well as all first order model generators behave much worse on the 3 -argument problems than on the corresponding 2-argument problems. As computing some output is a natural feature of a logic program, the advanced failure analysis is an important asset of our system.

Adding more sophisticated backtracking which does not fix the order of the cells in advance yields a substantial improvement on most problems. The system single $C S$ which sticks everywhere to the first conflict set is often the 
Table 3. Number of backtracks

\begin{tabular}{|c|c|c|c|c|c|c|c|c|}
\hline \multirow[t]{2}{*}{ Example } & \multirow{2}{*}{$\begin{array}{r}\text { naive } \\
\text { \#bckt }\end{array}$} & \multirow{2}{*}{\begin{tabular}{|r|} 
single CS \\
\#bckt
\end{tabular}} & \multicolumn{2}{|c|}{ best CS } & \multicolumn{2}{|c|}{\begin{tabular}{l|l} 
ABINDER \\
\end{tabular}} & SEM & \multirow{2}{*}{$\begin{array}{r}\mathrm{FMC}_{\text {ATINF }} \\
\text { \#bckt }\end{array}$} \\
\hline & & & \#bckt & total & \#bckt & \#bckt & \#bckt & \\
\hline appendlast & 41045 & 56 & 27 & 136 & 43 & 180 & 27 & 110019 \\
\hline reverselast & 41045 & 56 & 27 & 133 & 30 & 211 & 27 & 23445 \\
\hline nreverselast & $>10000$ & $>2000$ & 221 & 2426 & 190170 & $>10^{7}$ & 31285086 & $>?$ \\
\hline multiset1o & 4 & 3 & 3 & 11 & 4 & 4 & 3 & \\
\hline multiset 20 & 14 & 14 & 12 & 38 & 10 & 31 & 8 & 104 \\
\hline multiset3o & 127 & 75 & 76 & 122 & 33 & 75 & 86 & - \\
\hline blockpair2o & 9323 & 34 & $\overline{32}$ & 55 & 17 & 273 & 918 & 5567 \\
\hline blockpair3o & $>3000$ & 34 & 32 & 55 & 56 & 879 & 2904 & $>?$ \\
\hline blockpair2l & 32873 & 76 & 66 & 117 & 33 & 68 & 918 & 91404 \\
\hline blockpair3l & $>6000$ & 76 & 66 & 117 & 204 & 359 & 2904 & $>$ ? \\
\hline blockzero2o & 577 & 241 & 48 & 148 & 158 & 823 & 3495 & - \\
\hline blockzero3o & 1245 & 241 & 48 & 148 & 500 & 897 & 63032 & - \\
\hline blockzero2l & 1145 & 190 & 181 & 1044 & 98 & 1131 & 3415 & \\
\hline blockzero3l & 2289 & 190 & 181 & 1044 & 380 & 1123 & 63288 & \\
\hline blockzero2ls & 128926 & 21544 & 20284 & 31969 & 3615 & 3999226 & 201882 & \\
\hline tba & $>4000$ & 95369 & 41 & 91 & 72 & 23 & 5 & $\overline{33}$ \\
\hline grp & 19996 & 71 & 138 & 210 & 361 & 24 & 14 & - \\
\hline cl3 & 5 & 670 & 93 & 191 & 41 & 30 & 3 & \\
\hline
\end{tabular}

fastest, although it often needs more backtracks than best CS. It fails only on nreverselast which uses a 5 element domain and has a very large search space. However, on the equality problems it becomes obvious that a good choice of a conflict set is essential for solving such problems. In number of backtracks, best CS compares quite well with AB. Only on blockzero2ls it needs a lot more backtracks, while it needs a lot less on nreverselast. Perhaps on blockzero2ls, which has no solution, it suffers from the less optimal ordering because the search space has to be searched exhaustively.

From the model generators FINDER and SEM perform reasonably well in terms of time and also in number of backtracks. However, the results for FINDER were obtained only after a fine tuning of the different parameters and the representation of the problems (see [3]). The system also uses intelligent backtracking for deriving secondary conflict sets and some other forms of failure analysis. It has a smaller number of backtracks on the more complex planning problems than SEM. The system SEM is the fastest in raw speed and is not so sensible to the problem representation. Of the model generators, the system FMC $A T I N F$ is the weakest on the class of problems we consider. This result contrasts with the results in [7] where it is the best on several problems.

Compared with our system the model generators have to backtrack much more on the planning problems and the other logic programs where they have to explore the full space of interpretations while we look only for the least model of the program for a given pre-interpretation (the extra cost of evaluating the 
query in the least model is more than compensated for by the exponentially smaller search space). On the TPTP problems our system is doing worse which suggests that there is further room for making better use of the information in conflict sets.

\section{Conclusion}

In this paper we presented a method for proving failure of queries for definite logic programs based on direct execution of the abstracted program in XSBProlog, a standard top-down proof procedure with tabulation.

By using a better form of intelligent backtracking (proposed in [1]) which does not fix the enumeration order in advance and an improved failure analysis, we were able to compensate for the loss of flexibility which results from the direct execution of the abstracted program.

This way of intelligent backtracking could also be interesting for other systems, e.g. FMC $A T I N F$ of which Peltier reports that it is quite sensitive to the initial enumeration order.

While difference in speed with the $A B$ system are modest, the approach is still very interesting as the depth-first left-to-right execution results in a much better memory management so that larger problems can be tackled. The metainterpreter of the $\mathrm{AB}$ system keeps track of the whole top-down proof tree in evaluating the query, which leads to very large memory consumption.

Interesting future work is to further investigate some control issues. One could explore whether there is a good compromise between computing only one solution to the query and computing all solutions. One could try to further improve the backtracking by developing some heuristics which order a group of new elements when they are inserted in the ordered sequence.

\section{Acknowledgements}

We want to thank Kostis Sagonas for his help with the XSB system. Maurice Bruynooghe is supported by FWO-Vlaanderen. Nikolay Pelov is supported by the GOA project LP+.

\section{A Code for Some of the Problems}

\section{A.1 Multiset}

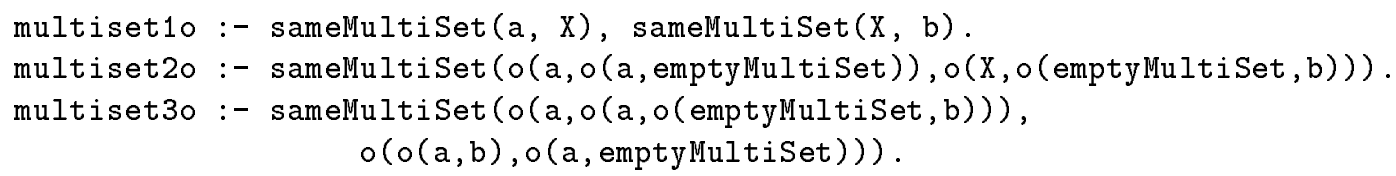


$\operatorname{sameMultiSet}(O(X, Y), o(X, Z)):-\operatorname{sameMultiSet}(Y, Z)$.

sameMultiSet $(o(O(X, Y), Z), U):-\operatorname{sameMultiSet}(o(X, o(Y, Z)), U)$.

sameMultiSet $(U, O(O(X, Y), Z)):-\operatorname{sameMultiSet}(U, O(X, O(Y, Z)))$.

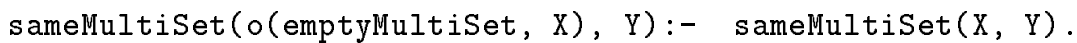

sameMultiSet $(X, o($ emptyMultiSet, $Y)):-\operatorname{sameMultiSet}(X, Y)$.

sameMultiSet $(O(X, Y), Z):-\operatorname{sameMultiSet}(O(Y, X), Z)$.

\section{A.2 Planning Problems}

Blocks are identified by integers represented as terms with the constant 0 and the function $s / 1$. The actionZero/3 predicate gives the possible actions and the causesZero $/ 3$ predicate tries to find a plan. In both predicates the first argument is the initial state, the last argument is the final state and the plan is collected in the second argument.

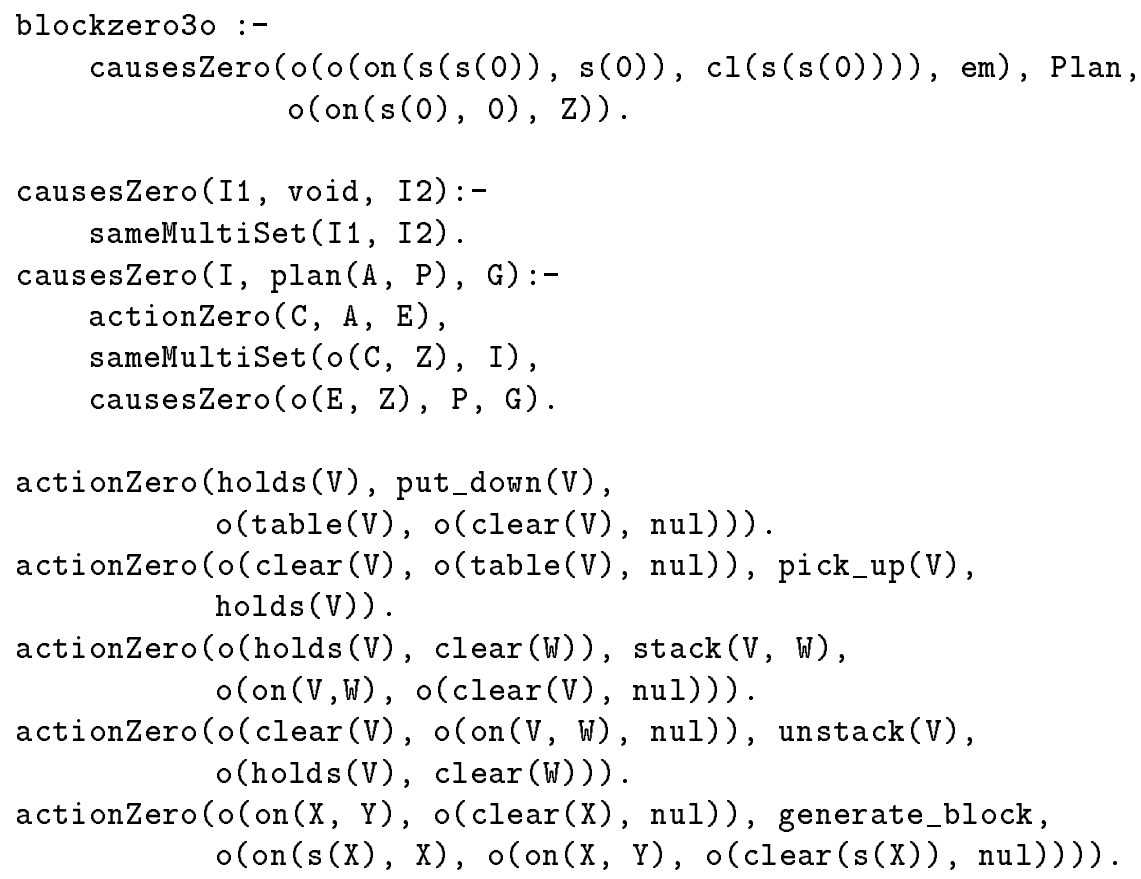

\section{References}

[1] M. Bruynooghe. Solving combinatorial search problems by intelligent backtracking. Information Processing Letters, 12(1):36-39, Feb. 1981.

[2] M. Bruynooghe, H. Vandecasteele, D. A. de Waal, and M. Denecker. Detecting unsolvable queries for definitive logic programs. In C. Palamidessi, H. Glaser, and K. Meinke, editors, Principles of Declarative Programming, 10th International Symposium, volume 1490 of Lecture Notes in Computer Science, pages 118-133. Springer Verlag, Sept. 1998. 
[3] M. Bruynooghe, H. Vandecasteele, D. A. de Waal, and M. Denecker. Detecting unsolvable queries for definitive logic programs. Journal of Functional and Logic Programming, 1999. To Appear.

[4] W. Chen and D. S. Warren. Tabled evaluation with delaying for general logic programs. Journal of the ACM, 43(1):20-74, Jan. 1996.

[5] M. Codish and B. Demoen. Analyzing logic programs using "PROP"-ositional logic programs and a magic wand. Journal of Logic Programming, 25(3):249-274, Dec. 1995.

[6] J. W. Lloyd. Foundations of Logic Programming. Springer-Verlag, second edition, 1987.

[7] N. Peltier. A new method for automated finite model building exploiting failures and symmetries. Journal of Logic and Computation, 8(4):511-543, 1998.

[8] J. A. Robinson. Automatic deduction with hyper-resolution. Int. Journal of Computer Math., 1:227-234, 1965.

[9] K. Sagonas, T. Swift, and D. S. Warren. XSB as an efficient deductive database engine. SIGMOD Record (ACM Special Interest Group on Management of Data), 23(2):442-453, June 1994.

[10] J. Slaney. Finder version 3.0 - notes and guides. Technical report, Centre for Information Science Research, Australian National University, July 1995.

[11] C. B. Suttner and G. Sutcliffe. The TPTP problem library (TPTP v2.1.0). Report AR-97-04, Fakultät für Informatik der Technischen Universität München, 1997.

[12] J. Zhang and H. Zhang. SEM: a system for enumerating models. In C. S. Mellish, editor, Proceedings of the Fourteenth International Joint Conference on Artificial Intelligence, pages 298-303, San Mateo, Aug. 1995. Morgan Kaufmann. 